\begin{abstract}
Drogenpolitik - ein altes/neues Thema
Bereits im Februar 1996 hat die FMH ihren Standpunkt zur Drogenpolitik unmissverständlich dargelegt (FMH und Drogenpolitik. SÄZ 9/1996). Damals wie heute ist es wichtig, im Bereich der psychoaktiven Substanzen eine umfassende Gesundheitspolitik zu gestalten, in die alle Probleme im Zusammenhang mit der Verwendung dieser Substanzen miteinbezogen werden. Der Wunsch nach einer drogenfreien Gesellschaft darf nicht verhindern, dass man sich pragmatisch mit der Realität der Existenz von Drogen und der Nachfrage danach auseinandersetzt!

Im Hinblick auf die kommende Abstimmung zur Hanfinitiative möchten wir mit dem nachstehenden Artikel die Diskussion in der Ärzteschaft ermöglichen.
\end{abstract}

Barbara Weil, Abteilung Prävention der FMH

\title{
Cannabispolitik: weder dämonisieren noch banalisieren
}

\author{
Aus fachlicher Perspektive erfordert eine nachhaltige Verbesserung der Can- \\ nabisproblematik politische Rahmenbedingungen, die es erlauben, chroni- \\ schem Cannabiskonsum wirksam und frühzeitig vorzubeugen. Dafür muss der \\ Konsum entkriminalisiert und der Handel in klare Bahnen gelenkt werden. Die \\ Annahme der «Hanf-Initiative», die voraussichtlich im Herbst zur Abstimmung \\ gelangt, macht das möglich.
}

\section{Robert Hämmiga , Jean-Félix Savary ${ }^{b}$ Markus Theunert ${ }^{c}$}

a Dr. med., Präsident der Schweizerischen Gesellschaft für Suchtmedizin (www.ssam.ch)

b Generalsekretär Groupement Romand d'Etudes des Addictions (www.grea.ch)

c Generalsekretär Fachverband Sucht (www.fachverbandsucht.ch)

Korrespondenz:

Dr. med. Robert Hämmig Universitäre Psychiatrische Dienste Bern

Funktionsbereich Sucht

Ziegelstrasse 7

CH-3010 Bern
Am 28. September oder 30. November dieses Jahres wird aller Voraussicht nach die Volksinitiative «Für eine vernünftige Hanfpolitik mit wirksamem Jugendschutz» (Hanf-Initiative) zur Abstimmung gelangen. Die Fachorganisationen der medizinischen und psychosozialen Berufe sind nun gefordert, «evidence-based» eine Position zu den Forderungen der Hanf-Initiative zu erarbeiten.

\section{Ziele}

Die Ziele einer «nüchternen», ideologiefreien Cannabispolitik können folgendermassen formuliert werden:

- Senkung der Konsumrate in der Bevölkerung;

- späterer Erstkonsum bei Jugendlichen;

- verminderte Schädlichkeit der konsumierten Produkte;

- frühzeitige Erkennung von und zielgruppengenaue Intervention bei gefährdeten Jugendlichen.

Die drei grossen Fachgesellschaften im Suchtbereich - die Schweizerische Gesellschaft für Suchtmedizin sowie die beiden sprachregionalen
Fachverbände GREA und Fachverband Sucht sind sich einig, dass eine Annahme der «HanfInititiave» die Erreichung dieser Ziele sehr viel realistischer macht als der heutige Status quo. Denn aus der Perspektive der Suchtfachleute ist die heutige Situation ein «worst case»-Szenario: Es gibt ein Gesetz, das Cannabis generell verbietet, das aber nicht umgesetzt werden kann, von Kanton zu Kanton verschieden gehandhabt wird, eine grosse Rechtsunsicherheit schafft, die Ressourcen in repressiven statt in präventiven Massnahmen bindet - und trotzdem ganz offensichtlich die Verbreitung von Cannabis nicht wirksam eindämmen kann!

In der Debatte zur Hanf-Initiative wird die Frage von besonderer Bedeutung sein, wie die populärwissenschaftlich viel zitierten «neuen» Studienergebnisse $\mathrm{zu}$ gewichten sind, die als Nachweis herangezogen wurden, dass Cannabis gefährlicher sei als bisher vermutet. Nachfolgend wird ausgeführt, warum eine kritische Sichtung dieser Ergebnisse diese Annahme nicht stützt. Dabei wird klar: Der regelmässige Konsum von Cannabis ist der Gesundheit - insbesondere von Adoleszenten - nicht zuträglich. Doch es gibt aus wissenschaftlicher Sicht keine wesent- 
lichen neuen Erkenntnisse, die ein generelles Verbot von Cannabis zusätzlich rechtfertigen würden. Indes: Die empirische Datenlage reicht aus, um von Cannabis als Rausch- und Genussmittel abzuraten. «Abraten» heisst allerdings nicht «verbieten» und «bestrafen», zumal wenig dafür spricht, dass die Bestrafung zu einer Reduktion des Konsums führt [1].

\section{Problemlast}

Cannabis ist die heute am meisten verbreitete illegale Droge in der Schweiz. 28 Prozent der Schweizer Bevölkerung im Alter von 15 bis 39 Jahren hat schon einmal im Leben Cannabis gebraucht (Gesundheitsbefragung 2002, BfS). Aktuelle epidemiologische Daten lassen annehmen, dass sich der Cannabiskonsum in der Schweiz auf hohem Niveau stabilisiert hat bzw. rückläufig ist [2].

\section{Generelles Risiko von Cannabis}

Die renommierte Zeitschrift «The Lancet» hat 2007 ein Expertenrating veröffentlicht [3], in dem 40 Forscher, Mediziner sowie Vertreter von Polizei und Justiz mit wissenschaftlichem Background aufgrund der vorliegenden evidenzbasierten Erkenntnissen die bekannten psychoaktiven Substanzen hinsichtlich folgender Faktoren einschätzten:

- gesundheitliche Gefahr für Konsumierende;

- Suchtpotential;

- Folgen des Konsums für die Gesellschaft.

Abbildung 1 zeigt, dass Cannabis von den 20 klassifizierten Substanzen an 11. Stelle des Risikoratings steht. Die Liste wird angeführt von Heroin, Kokain und Strassenmethadon. Die legalen Substanzen Alkohol und Tabak werden als

Abbildung 1

Expertenrating von 20 klassifizierten Substanzen.

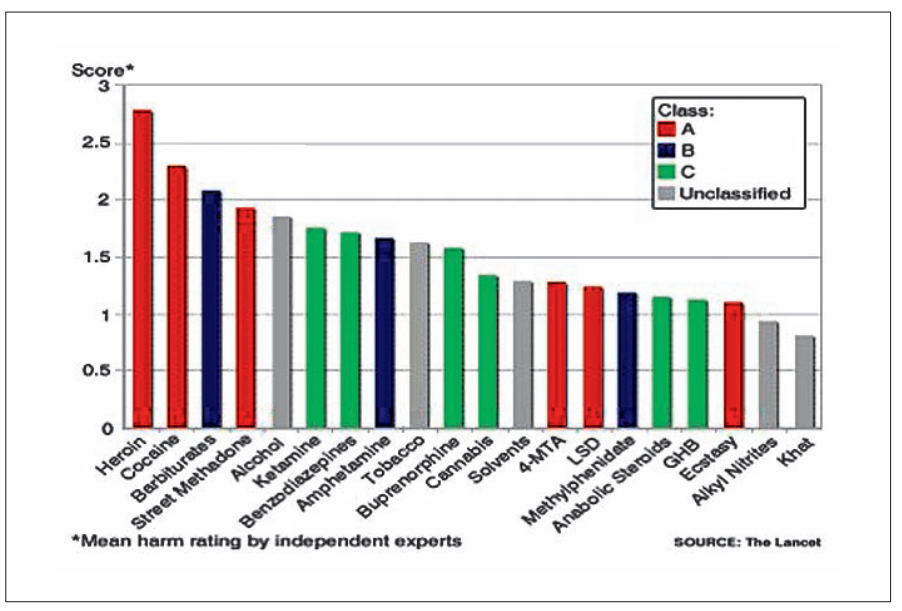

weitaus gefährlicher als Cannabis eingeschätzt, ebenso die Medikamentengruppe der Benzodiazepine und Amphetamine sowie Buprenorphin.

\section{Atemwege}

Häufiges Cannabisrauchen kann zu einer ganzen Anzahl von Lungenschäden wie chronischer Bronchitis und anderen Atemwegsentzündungen führen. Inwiefern diese durch das Tabakrauchen verursacht sind - in aller Regel wird Cannabis mit Tabak gemischt geraucht - oder durch die Wirkstoffe des Cannabis erzeugt werden, ist nicht völlig geklärt. Doch wer jahrelang regelmässig, insbesondere täglich, Cannabis konsumiert, muss mit Lungenschädigungen und Atembehinderungen rechnen [4].

Neuere Arbeiten kommen zum Schluss, dass bis heute keine schlüssige Evidenz für einen $\mathrm{Zu}$ sammenhang zwischen dem Cannabisrauchen und Lungenkarzinomen besteht [5]. Während der Tabakrauch klar krebserregende Bestandteile enthält, gibt es schwache Hinweise, dass bestimmte Cannabinoide antikarzinomatöse Aktivitäten besitzen [6]. Natürlich ist auch zu beachten, dass bei nichtrauchenden Teenagern mit einem regelmässigen Cannabiskonsum ein erhöhtes Risiko für Nikotinabhängigkeit besteht.

\section{Kognitive Entwicklung}

THC bewirkt zum Zeitpunkt des Konsums Wahrnehmungsveränderungen. Es beeinträchtigt das Reaktionsvermögen und das Kurzzeitgedächtnis. Klar ist, dass Menschen unter THC-Einfluss kein Fahrzeug lenken sollen.

Langzeitwirkungen auf die kognitive Leistungsfähigkeit wurden bisher vor allem bei intensivem, langjährigem Cannabiskonsum und nach dessen Beendigung untersucht. Hier resultieren eine Verminderung der Aufmerksamkeits-, Lern- und Kurzzeitgedächtnisleistungen.

Aber: Langzeiteffekte auf die kognitive Leistungsfähigkeit scheinen offenbar schon einen Monat nach der Abstinenz so geringfügig zu sein, dass sie nur noch sehr schwer messbar sind.

Relevant scheint allerdings das Einstiegsalter. Langzeitkonsumenten, die vor dem 17. Altersjahr mit dem Cannabiskonsum angefangen hatten, schnitten in den Testergebnissen schlechter $\mathrm{ab}$ als solche mit späterem Beginn.

\section{Motivation und Leistungsfähigkeit}

Viele Studien sind der Frage nachgegangen, ob sich Cannabiskonsum auf die Leistungsfähigkeit und Motivation von Jugendlichen auswirkt.

Die Studien kommen zum Schluss, dass es kaum Kausalbeziehungen zwischen Cannabiskonsum und psychosozialen Folgeschäden gibt 
[7]. Die Existenz eines «amotivationalen Syndroms» wird mehrheitlich bestritten. Eine neue Schweizer Studie zeigt, dass Jugendliche, die nur Cannabis konsumieren, besser sozial funktionieren als solche, die auch Zigaretten rauchen. Verglichen mit Abstinenten haben sie nicht häufiger psychosoziale Probleme [8].

\section{THC-Gehalt}

Der THC-Gehalt der gehandelten Cannabispflanzen ist gestiegen. In der Schweiz beträgt der durchschnittliche THC-Gehalt des Hanfkrauts etwa $13 \%$. International wird jedoch «nur» ein leichter Anstieg beobachtet. Der Konsumtrend weg von Hasch hin zu Gras macht die Vergleichbarkeit mit früheren Daten jedoch schwierig. Sicher ist: Es gibt «Zuchterfolge» von Pflanzen mit sehr hohem THC-Gehalt bis 30 Prozent. THC-Gehalt und Gefährdung stehen aber nicht in einem direkten Zusammenhang.

\section{Abbildung 2}

Momentan die am meisten konsumierte illegale Droge in der Schweiz: Cannabis.

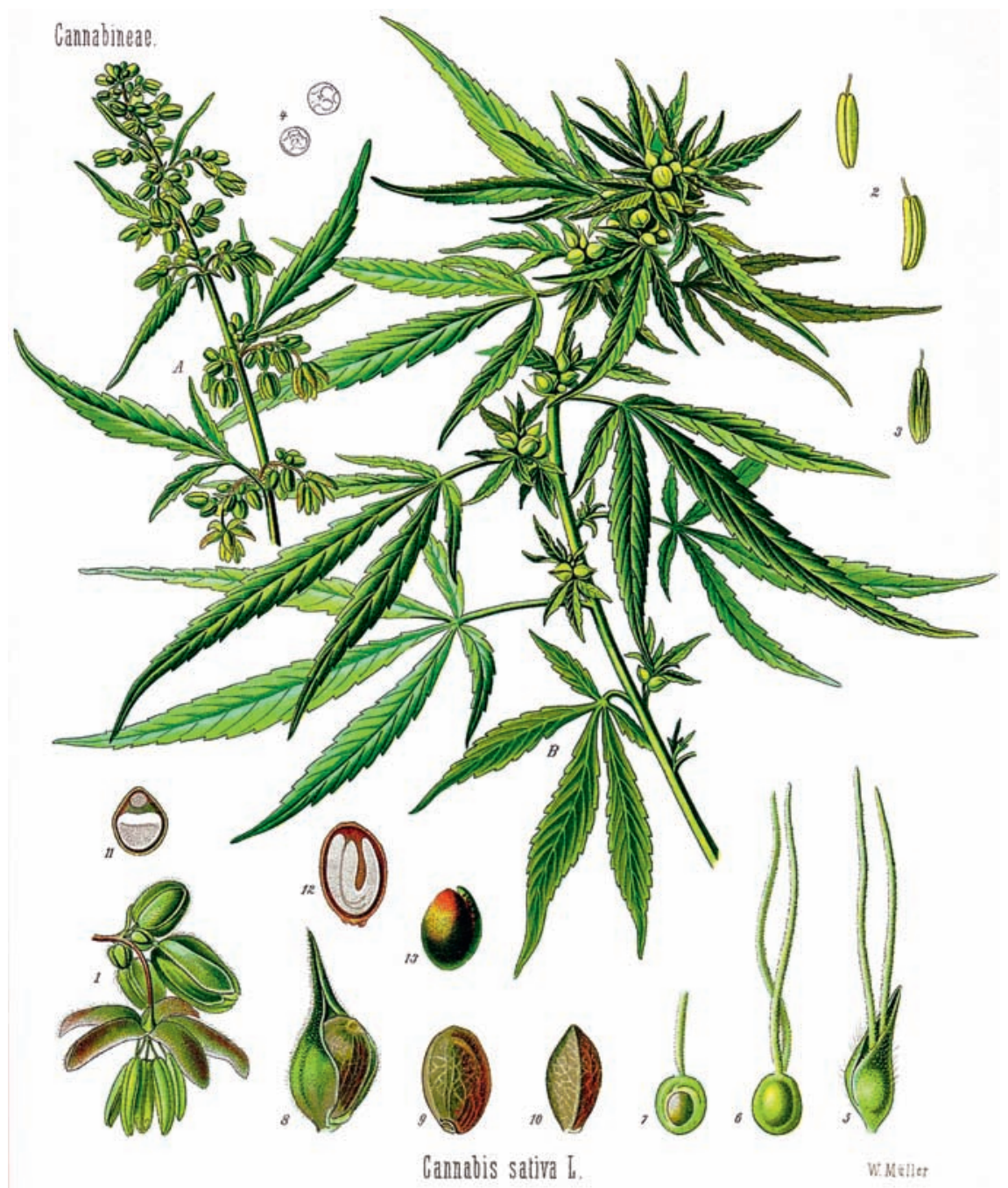

In den Basalganglien, der Hirnrinde und dem Hippokampus gibt es Anandamidrezeptoren, an die das THC andocken kann. Durch ein Andocken im Kleinhirn und den Basalganglien wird die Motorik beeinflusst, Zeitgefühl und Konzentrationsfähigkeit werden durch ein Anheften an Rezeptoren in der Hirnrinde verändert und durch Ankoppelung an Rezeptoren des Hippokampus werden Gedächtnisleistung und Informationsverarbeitung beeinflusst. Der erste körpereigene Stoff, der an die Anandamidrezeptoren ankoppelt, das Anandamid, von dem der Rezeptortyp auch seinen Namen hat, wurde erst 1992 entdeckt.

Da der Hirnstamm, der lebenswichtige Funktionen wie die Atmung steuert, kaum solche Rezeptoren besitzt, sind lebensbedrohliche Cannabisvergiftungen so gut wie ausgeschlossen. Bis heute ist kein Fall bekannt, in dem ein Mensch direkt durch die Wirkung von Cannabis gestorben ist. Deshalb hinkt auch der Vergleich mit Alkohol: Während 40prozentiger Schnaps tatsächlich eine $8 \mathrm{mal}$ höhere Wirkung und Schädlichkeit als 5 prozentiges Bier hat, stimmt dieser Zusammenhang beim THC nicht.

Die gestiegene THC-Konzentration führt möglicherweise bei cannabisunerfahrenen Menschen zu unangenehmen Rauscherfahrungen (die allenfalls «Probierer» von weiterem Konsum abhalten können). Cannabiserfahrene Konsumenten passen das Rauchverhalten bzw. das Mischverhältnis von Cannabis und Tabak dem THC-Gehalt an; sie erreichen mit weniger Cannabis und mithin auch mit geringerer Menge von Tabak die gewünschte Rauschwirkung.

\section{Abhängigkeitspotential}

THC kann Abhängigkeiten verursachen. Schwere Entzugserscheinungen sind aber nicht bekannt. Der Konsum von Cannabis kann jederzeit ohne grössere körperliche Entzugserscheinungen eingestellt werden.

\section{Cannabis und Depressionen}

Zwar besteht keine Einigkeit in den Befunden, doch neuere prospektive Studien zeigen mögliche Zusammenhänge zwischen Cannabiskonsum und späteren Depressionen. Besonders bei Mädchen, die Cannabis bereits früh konsumieren, besteht ein erhöhtes Risiko für spätere Depressionen [9].

\section{Cannabis und Schizophrenien}

Cannabiskonsum kann eventuell den Krankheitsausbruch beschleunigen und den Krankheitsverlauf negativ beeinträchtigen [10]. Mehrere neue Untersuchungen versuchen aufzu- 
zeigen, dass ein regelmässiger Cannabiskonsum mit erhöhtem Psychoserisiko einhergeht. Eine Zürcher Studie versuchte nachzuweisen, dass die erhöhte Neueintretensrate männlicher Jugendlicher in psychiatrische Einrichtungen wegen psychotischer Symptome in den 90er Jahren tendenziell mit dem Anstieg des Cannabiskonsums in dieser Zeit zusammenfällt, und postulierte eine Kausalität [11]. Bei jungen Frauen (die übrigens ihren Konsum mehr steigerten) ergab sich in dieser Studie keine solche Tendenz. Die These einer kausalen Beziehung zwischen Cannabiskonsum und Auslösung von Schizophrenien ist damit nicht erwiesen. Diese Aussage wird auch gestützt durch Befunde aus Australien. In Australien nahm in den letzten 30 Jahren die Prävalenz des Cannabiskonsums massiv zu, jedoch blieben die Inzidenzraten für Schizophrenien davon über den ganzen Zeitraum unbeeinflusst [12]. Inzidenzraten schizophrener Neuerkrankungen können in allen Gesellschaften über kurze Zeiträume erheblich schwanken. Unter den vielen Einflüssen, die diese Raten beeinflussen, gelten soziokulturelle Bedingungen allgemein als erwiesen (s. z. B. Übersicht bei [13]) und die gesellschaftlichen Bedingungen für die Jugendlichen veränderten sich im beobachteten Zeitraum in der Schweiz stark (Jugendarbeitslosigkeit!). Zu erwähnen ist, dass unter Schizophrenen gegenüber der Normalbevölkerung die Prävalenz für Suchtmittelmissbrauch generell stark erhöht ist, insbesondere auch für Alkohol und Tabak. Die Verbindung von Sucht und Schizophrenien konnte bis heute noch nicht vollständig entschlüsselt werden [14].

\section{Generalpräventive Wirkung des Verbots}

Es liegen keine neuen Befunde vor, die belegen, dass die Cannabisprohibition die Prävalenz des Konsums zu senken vermag. Der internationale Vergleich zeigt keinen Zusammenhang zwischen strengen gesetzlichen Regelungen und tiefen Konsumraten [15].

\section{Politische Würdigung}

Cannabis ist keine harmlose Substanz. Während den meisten Konsumierenden ein risikoarmer Umgang gelingt, entwickelt gemäss internationalen Erfahrungen etwa jede/r zwanzigste ein problematisches Konsummuster. Eine fachlich fundierte Cannabispolitik muss es ermöglichen, diesem Segment frühzeitig die angemessene Behandlung zukommen zu lassen. Ein vollständiges Cannabisverbot wirkt dabei jedoch erschwerend, weil die Behandlungsschwelle durch die Illegalität erhöht wird.
Die Cannabisprohibition hat weitere unerwünschte Nebeneffekte:

- Das Cannabisverbot verunmöglicht eine Kontrolle der konsumierten Produkte. Die Konsumierenden sind nicht geschützt. Ihnen fehlen Informationen darüber, was sie konsumieren;

- der THC-Gehalt ist nicht beschränkbar;

- giftige Pestizide werden mitinhaliert;

- Beimischungen wie Glassplitter stellen eine Gefährdung für die Konsumierenden dar;

- die Märkte für Cannabis, Kokain und Heroin vermischen sich;

- der Schwarzmarkt wird zunehmend durch internationale kriminelle Netzwerke beherrscht, die lokale Hersteller und Händler verdrängen;

- Jugendliche oder erwachsene Problemkonsumenten können nicht ohne Angst vor Sanktionen über ihre Probleme sprechen: Der Cannabiskonsum wird versteckt, eine frühzeitige Erkennung verhindert und die Gefahr vergrössert, dass der Konsum chronisch wird;

- mehr als 30000 Verzeigungen wegen Cannabis pro Jahr (gemäss Fedpol-Statistik sind $10 \%$ der Verzeigten minderjährig, 20\% über 35 jährig) verursachen einen enormen administrativen Leerlauf und produzieren unnötige Millionenkosten. Dieses Geld fehlt für Prävention und Beratung.

Unter dem Strich ist aus fachlicher Perspektive die Einschätzung eindeutig: Die Bilanz der heutigen Verbotspolitik ist deutlich negativ. Das Verbot verursacht wesentlich mehr Schäden, als es verhindert (s. auch [16]). Eine Entkriminalisierung des Konsums und die Überführung des Schwarzmarkts in einen klar regulierten Markt (z.B. über Apotheken) sind aus medizinischer Sicht zu begrüssen. Die Annahme der HanfInitiative ermöglicht die Erarbeitung einer praktikablen Gesetzesgrundlage, die die Cannabisproblematik wirklich anpackt, statt sie nur zu verwalten. Ohne Ideologie. Ohne Banalisieren. Mit Augenmass.

\section{Literatur}

1 Schaub M, Stohler R. Langzeitschäden durch Cannabis - ein unterschätztes Problem? Schweiz Med Forum. 2006;6(50):1129-32.

2 Schmid H, Delgrande Jordan M, Kuntsche EN, Kündig H, Annaheim R. Der Konsum von psychoaktiven Substanzen von Schülerinnen und Schülern in der Schweiz. Forschungsbericht der Schweizerischen Fachstelle für Alkohol- und andere Drogenprobleme. Lausanne: SFA; 2007. 
3 Nutt D, King L, Saulsbury W, Blakemore C. Development of a rational scale to assess the harm of drugs of potential misuse. Lancet. 2007; 369(9566):1047-53.

4 Beshay M, Kaiser H, Niedhart D, Reymond MA, Schmid RA. Emphysema and secondary pneumothorax in young adults smoking cannabis. Eur J Cardiothorac Surg. 2007;32(6):834-8.

5 Mehra R, Moore BA, Crothers K, Tetrault J, Fiellin DA. The association between marijuana smoking and lung cancer. A systematic review. Arch Intern Med. 2006;166(13): 1359-67.

6 Hashibe M, Morgenstern H, Cui Y, Tashkin DP, Zhang ZF, Cozen W, et al. Marijuana use and the risk of lung and upper aerodigestive tract cancers: results of a population-based case-control study. Cancer Epidemiol Biomarkers Prev. 2006;15(10): 1829-34.

7 Macleod J, Oakes R, Copello A, Crome I, Egger M, Dickman M. Psychological and social sequelae of cannabis and other illicit drug use by young people: a systematic review of longitudinal, general population studies. Lancet. 2004;363(9421): 1579-88.

8 Suris JC, Akre C, Berchtold A, Jeannin A, Michaud PA. Some go without a cig: Characteristics of cannabis users who have never smoked tobacco. Arch Pediatr Adolesc Med. 2007;161(11):1042-7.

9 Copeland J. Cannabis use, depression and public health. Addiction. 2006;101(10):1380.

10 Bühler B, Hambrecht M, Löffler W, an der Heiden W, Häfner H. Precipitation and determination of the onset and course of schizophrenia by substance abuse - a retrospective and prospective study of 232 population-based first illness episodes. Schizophr Res. 2002;54(3):243-51.
11 Ajdacic-Gross V, Lauber C, Warnke I, Haker H, Murray RM, Rössler W. Changing incidence of psychotic disorders among the young in Zurich. Schizophrenia Research. 2007;95(1):9-18.

12 Degenhardt L, Hall W, Lynskey M. Testing the hypotheses about the relationship between cannabis use and psychosis. Drug Alcohol Depend 2003; 71(1):37-48.

13 Nasrallah HA, Smeltzer DJ. Contemporary diagnosis and Management of the patient with schizophrenia. Newtown, PA: Handbooks in Health Care Co.; 2003.

14 Gregg L, Barrowclough C, Haddock G. Reasons for increased substance use in psychosis. Clin Psychol Rev. 2007;27(4):494-510.

15 Reinarman C, Cohen PD, Kaal HL. The limited relevance of drug policy: cannabis in Amsterdam and San Francisco. Am J Public Health. 2004;94(5): 836-42.

16 Grotenhermen F. The toxicology of cannabis and cannabis prohibition. Chem Biodivers. 2007;4(8): 1744-69.

- Arnaud S, Zobel F, Gervasoni JP, Schnoz D, Dubois-Arber F, Isenring G-L, et al. Monitoring de la problématique du cannabis en Suisse. Etude sentinelle 2004-2006. Raisons de Santé, 127b. Lausanne: Institut universitaire de médecine sociale et préventive; Institut de criminologie et de droit pénal de l'Université de Lausanne; 2007.

- Linskey MT. Life-time cannabis use and late onset mood and anxiety disorders. Addiction. 2007; 102(8):1181-2. 